\title{
Beliefs in COVID-19 conspiracy theories, compliance with the preventive measures, and trust in government medical officials
}

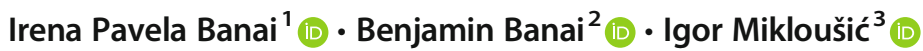 \\ Accepted: 19 May 2021 / Published online: 26 May 2021 \\ (C) The Author(s), under exclusive licence to Springer Science+Business Media, LLC, part of Springer Nature 2021
}

\begin{abstract}
The COVID -19 pandemic represents a global health crisis, so adherence to government guidelines and public health advice is critical in reducing transmission rates. Despite this, it has been reported that a minority of people do not comply with the governmental guidelines. When considering the reasons why some people do not comply with preventive measures, previous studies have shown that beliefs in COVID-19 conspiracy theories negatively predict responsible pandemic-related behaviour. This, in turn, could seriously undermine success in combating the pandemic. Our aim was, therefore, to further investigate the relationship between conspiracy beliefs and adherence to official COVID-19 medical guidelines by including mediating roles of beliefs in pseudoscientific information and trust in government officials. A total of 1882 adults from Croatia provided sociodemographic information and completed several scales related to COVID-19 conspiracy beliefs, beliefs in pseudoscientific information, trust in government officials, and adherence to official COVID-19 guidelines. A multiple mediation analysis revealed a direct negative effect of COVID-19 conspiracy beliefs on compliance with the preventive measures. In addition, conspiracy beliefs were indirectly related to compliance via trust in government officials. The present study builds upon emerging research showing that conspiracy beliefs may have significant social consequences and pose a potential risk to public health. Practical implications of these findings are discussed further.
\end{abstract}

Keywords COVID-19 $\cdot$ Public health $\cdot$ Conspiracy theory $\cdot$ Preventive measures

\section{Introduction}

The COVID-19 pandemic caused by coronavirus represents a global health crisis, and up until second half of April 2021 the pandemic has caused or is associated with almost three million deaths worldwide (World Health Organisation, 2021a). In Croatia, where the present study was conducted, 323,036

Irena Pavela Banai

ipavela@unizd.hr

Benjamin Banai

benjamin.banai@gmail.com

Igor Mikloušić

igor.miklousic@pilar.hr

1 Department of Psychology, University of Zadar, Obala k. Petra Krešimira IV, 2, 23000 Zadar, Croatia

2 Banai Analitika, Zadar, Croatia

3 Institute of Social Sciences Ivo Pilar, Zagreb, Croatia people had tested positive for coronavirus and 6905 have died as of April 26th, 2021 (Croatian Institute of Public Health, 2021). At the very beginning of the pandemic, the Croatian government responded quickly by issuing evidence-based prevention guidelines and numerous restrictions, such as enforcing social distancing measures, closing public transport, schools, restaurants, shopping centres and other public places such as cinemas and libraries. Confirmed coronavirus cases and people suspected of being infected (those who were in close physical contact with infected individuals) were ordered 14-day quarantine, which has left tens of thousands of people in self-isolation.

Although compliance with government guidelines and public health recommendation is crucial in reducing transmission rates during the COVID-19 pandemic, it has been reported that a minority of people do not comply with the governmental guidelines. The Ministry of Interior of the Republic of Croatia reported that by mid May 2020, nearly two thousand potentially infected individuals intentionally disregarded instructions to self-isolate, increasing the possibility of spreading the virus. When considering the reasons why some people 
do not follow self-isolation instructions, it is worth noting that different socio-demographic factors have been shown to play a significant role. For example, Atchison et al. (2021) reported that adoption of social-distancing measures was higher among people aged 70 and over compared to younger adults, possibly because they are the most vulnerable group. Furthermore, people with low household incomes, and ethnic minority groups were less likely to self-isolate. In addition, Zhang et al. (2020) found that married people were more likely to follow self-isolation measures. This study also showed that education level was positively associated with the willingness to self-isolate, although others did not find this effect (Plohl \& Musil, 2021).

Aside from socio-demographic factors related to compliance with the health guidelines, previous studies have shown that different psychological variables predict pandemicrelated behaviours. For example, Plohl and Musil (2021) found that COVID-19 risk perception and trust in science predicted compliance with COVID-19 preventive measures. When considering trust in science, it is important to take into account a wide and rapid spread of various conspiracy theories and misinformation related to the current pandemic. DirectorGeneral of the World Health Organization noted that experts are now fighting an infodemic along with the epidemic, as it is clear that belief in COVID-19 conspiracy theories could potentially influence people's behaviour and increase distrust in governmental preventive measures.

In modern society, conspiracy theories are a widespread phenomenon that can be defined as a belief that a group of people secretly works to achieve some malevolent goal (Bale, 2007). An important aspect of conspiracy theories is that they are more likely to occur during social crisis (van Prooijen \& Douglas, 2017), and COVID-19 pandemic, with global health, political, and economic consequences, represents such a situation.

The importance of examining how belief in conspiracy theories relates to people's behaviour during the COVID-19 pandemic is demonstrated by studies conducted prior to the COVID-19 pandemic, which have shown that agreement with conspiracy theories was associated with disregarding public health advice in several areas. For example, Jolley and Douglas (2014a) found a negative relationship between antivaccine conspiracy beliefs and vaccination intentions. In addition, participants exposed to climate change conspiracy theories report a decreased intention to reduce their carbon footprint (Jolley \& Douglas, 2014b). Regarding the relationship between epidemic-related conspiracy beliefs and behaviour, studies have shown that the likelihood of having unsafe sex is higher among AIDS conspiracy believers (Bogart \& Thorburn, 2005; Grebe \& Nattrass, 2012).

These findings imply that belief in conspiracy theories may pose a threat to public health and highlight the importance of investigating them further in the context of the ongoing pandemic. The content and characteristics of conspiracy theories about COVID-19 are not much different from those related to previous epidemics (e.g., Zika virus, Wood, 2018). Most theories include the belief that the coronavirus is manmade virus created to control population, possibly via $5 \mathrm{G}$ technology or global vaccination. Theories also include the belief that COVID-19 is no more dangerous than regular flu because mortality rates are lower than those reported by government officials and health care providers. Research on how conspiracy beliefs relate to the behaviour has shown that people high in conspiracy mentality are less likely to comply with preventive behaviours recommended by the government (Marinthe et al., 2020). Furthermore, Allington and Dhavan (2020) found a negative relationship between belief in COVID-19 conspiracy theories and compliance with public health recommendations. In the same study, $37 \%$ of participants who reported agreement that $5 \mathrm{G}$ technology was associated with COVID-19 also believed that there was no good reason for the lockdown. Moreover, Erceg et al. (2020) showed that endorsement of COVID-19 unfounded beliefs led to less responsible behaviour. Similar findings were reported by Teovanović et al. (2020). Moreover, the belief that COVID-19 was a hoax was found to negatively predict containment-related behaviours, such as hand washing and maintaining physical distance (Imhoff \& Lamberty, 2020) or face mask wearing and social distancing (Hornik et al., 2021). In a similar vein, Freeman et al. (2020) concluded that coronavirus conspiracy beliefs were associated with lower adherence to all government guidelines. Furthermore, other researchers found that COVID-19 conspiracy beliefs were negatively associated with adherence to social distancing guidelines (Biddlestone et al., 2020; Bierwiaczonek et al., 2020). It has been also reported that the COVID-19-related conspiracy beliefs were inversely related to the taking of preventive actions, including wearing a face mask and intention to be vaccinated against COVID-19 (Romer \& Jamieson, 2020). However, some studies have produced inconsistent results. For example, Čavojová et al. (2020) and Alper et al. (2020) found no correlation between coronavirus specific conspiracy beliefs and recommended behaviour against COVID-19, which calls for further, more nuanced exploration of this subject.

Aside from widespread conspiracy beliefs about the origin of coronavirus and false mortality rates, people are exposed to many pseudoscientific medical recommendations to prevent and cure the infection. Some of these recommendations can be dangerous and even lethal, like ingesting disinfectant or exposing the body to UV light. Less dangerous but equally ineffective methods of preventing or curing the infection found circulating online include consumption of garlic, colloidal silver, high doses of vitamin C, drinking water every $15 \mathrm{~min}$, and drinking hot beverages. The fact that people actually turn to unscientific remedies during pandemic is supported by several 
extreme cases. For example, the South China Morning Post reported that a woman from Tiantai County ate up to $1.5 \mathrm{~kg}$ of garlic in hopes of preventing the coronavirus infection (Yan, 2020). She then suffered consequences such as throat inflammation and inability to speak. An even more extreme case comes from Iran, where more than 700 people died from methanol poisoning after trying to prevent infection by ingesting toxic alcohol (Al-Arshani, 2020). In related news, Belgium Poison Control Center reported a $15 \%$ increase in hazardous substances poisoning calls during pandemic (Belgium Poison Control Center, 2020). In addition, according to the report from American Centers for Disease Control and Prevention, $39 \%$ of participants engaged in dangerous practices to prevent coronavirus transmission, including washing food with bleach, applying disinfectants to the skin, and even drinking diluted bleach solutions (Gharpure et al., 2020). Previous studies have shown an association between conspiracy beliefs and endorsement of pseudoscientific medical treatments. For example, Lamberty and Imhoff (2018) reported that conspiracy mentality predicted a preference for alternative over biomedical therapies. In the context of the current pandemic, we assumed that belief in COVID-19 conspiracy theories would be positively related to endorsement of pseudoscientific medical information, which in turn could lead to ignoring government pronouncements.

When it comes to government pronouncements, they can be seriously undermined by conspiracy theories. As Connolly et al. (2019) noted, conspiracy theories can be viewed as a marker of institutional distrust. In other words, people who endorse conspiracy beliefs are more likely to doubt government communication. In the context of the current pandemic, belief in COVID-19 conspiracy theories was found to decrease institutional trust and support for government regulations (Pummerer et al., 2021). Furthermore, belief in COVID conspiracy theories was associated with less trust in the local government (Earnshaw et al., 2020).

\section{The Present Study}

Based on previous studies, the spread of conspiracy theories can potentially lead to disregard of official medical recommendations and government restrictions. In this study, we propose that this relationship is mediated by two additional factors. First, given that conspiracy beliefs predict endorsement of medical misinformation (Lamberty \& Imhoff, 2018), we propose that pseudoscientific information beliefs may be an important mediator between conspiracy beliefs and adherence to COVID-19 guidelines. Second, considering that conspiracy theories can undermine authoritative information and government orders, belief in conspiracy theories could lower trust in government officials and thus lead to lower compliance with government restrictions.
Therefore, in the present study, we aimed to investigate the predictive power of conspiracy theories related to COVID-19 in explaining the level of compliance with official COVID-19 recommendations, by including mediating role of pseudoscientific information beliefs and trust in government officials. In this way, this study further explores the mechanisms by which conspiracy beliefs can lead to undesirable social and medical outcomes. In general, the present study builds upon emerging research showing that conspiracy beliefs may represent a substantial public health risk.

\section{Method}

\section{Participants}

The minimum required sample size was determined using Monte Carlo power analysis designed for multiple mediation models (Schoemann et al., 2017). Following previous studies (e.g., Erceg et al., 2020; Marinthe et al., 2020) we assumed small to moderate correlations between variables in the model. We estimated the minimum sample size needed to achieve a power of 0.80 , with confidence level of $99 \%$ using 10,000 Monte Carlo replications with 20,000 draws. Power analysis indicated that the sample size for this research setting should be at least 650 .

Croatian citizens aged $18+$ years were invited to participate in the online study. Therefore, being a Croatian citizen and older than 18 years of age were main eligibility criteria for taking part in this study. Participants were recruited through advertisements on social media websites, especially Facebook. The invitation to participate in the study was also published by Index.hr., the most popular news website in Croatia according to SimilarWeb (2020). Therefore, convenience and snowball sampling was used. In total, 1976 participants completed all measures in the study. We oversampled for two reasons. First, all measures used in this study were novel and developed for the purpose of this study. Therefore, a large sample size was necessary to conduct factor analyses. Second, we considered the possible exclusion of some participants who did not meet the study requirements. In this regard, we excluded 94 participants who failed the three attention check questions that explicitly asked them to mark the highest value ("This is a control question. Please mark the highest value on the right"). The resulting sample consisted of 1882 participants (1268 women, 596 men, 18 responded as "Do not wish to answer"; $M_{\text {age }}=36.57, S D_{\text {age }}=11.18$ ).

\section{Measures}

Participants were asked to provide sociodemographic information and to complete several scales related to COVID-19 conspiracy beliefs, pseudoscientific information beliefs, trust 
in government officials, and compliance with official COVID19 guidelines. All scales were developed for the purpose of this study. Therefore, the factor structures of the responses to the new measures were reported in the Results section, while all items and their factor loadings are presented in the online Supplementary materials.

\section{Sociodemographic Information}

Participants were asked to provide information about their gender (man, woman, prefer not to say), age, and highest education level (from primary education to doctorate).

\section{COVID-19 Conspiracy Beliefs}

To assess COVID-19 conspiracy beliefs, we created a list of nine items, each describing one of the popular conspiracy theories about the origin of the coronavirus (e.g., "Coronavirus was intentionally made in a laboratory", "Coronavirus was created as a bioweapon in the war between the powerful countries (like China, USA, Russia)"), its spread ("Spread of the coronavirus is related to the $5 G$ technology", "Bill Gates is using the pandemic for population control"), threat level (e.g., "Coronavirus is equally or less dangerous than the regular flu", "Videos and photographs of empty hospitals are a proof that the pandemic is a hoax"), and infection and mortality rates ("Official infection and mortality rates provided by the WHO are false"). Items included in the COVID-19 conspiracy beliefs scale represented the most prevalent conspiracy theories widely shared at the beginning of the pandemic. Participants indicated agreement with each statement on a scale ranging from 1 (strongly disagree) to 5 (strongly agree).

The average rating on a 5-point scale on these nine items was used as the level of endorsement of the COVID-19 conspiracy beliefs.

\section{Pseudoscientific Information Beliefs}

To assess pseudoscientific information beliefs, we used a list of products frequently presented as effective cures for COVID-19 on social media, without any scientific evidence. We have also compared our observations of fake news about the products to WHO Mythbusters (World Health Organisation, 2021b) and used it to further support and expand our list. We asked participants to rate their agreement that the following products can prevent/cure the coronavirus infection: alcohol, garlic, high doses of vitamin $\mathrm{C}$, colloidal silver, and hot beverages. Each product was rated on a 5-point scale, ranging from 1 (strongly disagree) to 5 (strongly agree).

\section{Trust in Government Officials}

Government officials who provided information and guidelines to the public during the pandemic in Croatia were represented by the National Civil Protection Headquarters, which was the prime communicator for COVID numbers, information, and restrictions. To assess trust in government officials, we asked participants to report their agreement with each of the five newly constructed statements about the work of National Civil Protection Headquarters (e.g., "I trust the National Civil Protection Headquarters and I think they are doing a good job", "Members of the National Civil Protection Headquarters are experts in their field", "National Civil Protection Headquarters works in the best interest of the Croatian people"). Participants reported their agreement with each statement on a scale from 1 (strongly disagree) to 5 (strongly agree).

\section{Compliance with Official COVID-19 Guidelines}

Participants were asked to indicate their compliance to eight behaviours that represented the most important guidelines communicated by government officials during the pandemic. Therefore, items included in this newly constructed scale reflect main guidelines communicated to the public by the official government communications at that time. The behaviours included physical-distancing measures (e.g., "I keep physical distance of at least two meters in enclosed spaces and at least one meter in the open", "I avoid crowded places", "I avoid meeting with my friends"), and increased hygiene measures (e.g., "I wash/disinfect my hands regularly", "I sneeze into my elbow"). Participants reported how often they acted according to the COVID-19 prevention guidelines by using a 5 -point scale ranging from 1 (never) to 5 (always).

\section{Procedure}

Ethical approval was obtained from the Ethics Committee of the Department of Psychology at the University of Zadar. The study was set online by using Google Form. Data were collected between the 15th and 26th of May 2020. Prior to the start of the survey, participants were guaranteed anonymity of their responses and presented with a general instruction stating that the aim of the study was to investigate attitudes towards the COVID-19 pandemic, and some aspects of pandemicrelated behaviour. The instruction also included the estimated study duration of $15 \mathrm{~min}$. Following the instructions, participants were asked to provide digital informed consent before being directed to the scales described above.

Upon completion of the study, participants were given the opportunity to leave their personal contact and enter a prize draw to win 200 Croatian kuna. To ensure the anonymity of their responses, participants were instructed to follow a link 
that directed them to a separate Google form where they could leave their contact information. This way, their responses could not be linked to their personal data.

\section{Statistical Analyses}

The analyses were conducted using $R$ ver. 4.0.1. (R Core Team, 2020), using packages psych v. 1.9.12 (Revelle, 2019), paran (Dinno, 2018) and lavaan v. 0.6-6 (Rosseel, 2012). To explore the underlying structure of the new measures, COVID-19 conspiracy beliefs, Pseudoscientific information beliefs, Trust in government officials, and Compliance with official COVID-19 guidelines, we conducted exploratory and confirmatory factor analyses. First, we randomly split the dataset into two partitions, each consisting of $n=941$ participants. The first partition was used to conduct exploratory factor analysis (EFA), and the other was used to validate the obtained factor structure using confirmatory factor analysis (CFA). Factorability of the EFA data partition was assessed using the Kaiser-Meyer-Olkin measure of sampling adequacy (KMO) and Bartlett's sphericity test. Values of KMO greater than 0.80 and a statistically significant Bartlett's sphericity test indicated that the data were appropriate for the application of factor analysis. Furthermore, we decided on the number of factors to retain based on the results of parallel analysis (Horn, 1965) of 10,000 randomly drawn samples using the $R$ package paran v. 1.5.2 (Dinno, 2018). paran produces eigen values adjusted for sample size error-induced inflation, and it is recommended to retain factors with adjusted eigenvalues greater than 0 (Dinno, 2014). Each parallel analysis was conducted using 10,000 random samples and by comparing the eigenvalues of the factors from the actual data to the eigenvalue value of 95 th percentile of random data. If the parallel analysis indicated that more than one factor should be retained, Promax rotation was performed after EFA. In addition, items with loadings greater than 0.32 were considered valid measures of a factor (Tabachnick \& Fidell, 2012). The EFA was followed by the CFA, in which a factor structure was specified according to the exploratory analysis. The CFA models were estimated using the maximum likelihood procedure, and root mean square error of approximation (RMSEA, Steiger, 1990), Tucker-Lewis index (TLI, Tucker \& Lewis, 1973), standardized root mean square residual and comparative fit index (SRMR and CFI, Bentler, 1990) as fit indices were used to evaluate model fit. Good model fit is characterized by low RMSEA and SRMR (both $<0.06$ ) and high values of TLI and CFI (both >0.95). All factor loadings for the items of the novel measures can be found in the online Supplementary materials.

After examining the correlations between pairs of variables, we conducted a multiple mediation analysis to examine the mediating role of pseudoscientific information beliefs and trust in government officials on the relationship between
COVID-19 conspiracy beliefs and compliance with official COVID-19 guidelines as an outcome variable. Multiple mediation analysis was conducted using $\mathrm{R}$ package lavaan $\mathrm{v}$. 0.6-6 (Rosseel, 2012) with maximum likelihood estimator and standard errors were calculated using 1000 bootstrap samples. The significance of the effects was evaluated by inspecting the $p$-values and $95 \%$ confidence interval (CI) of the parameters. Finally, we estimated the power of indirect effects using Monte Carlo-based post-hoc power analysis (Schoemann et al., 2017).

\section{Results}

\section{Factor Analyses}

\section{COVID-19 Conspiracy Beliefs}

Both measures of factorability, KMO and Bartlett's sphericity test, indicated that the correlation matrix of nine COVID-19 conspiracy beliefs items is suitable for analysis $(\mathrm{KMO}=0.92$, Bartlett's test: $\left.\chi^{2}(36)=5845.99, p<0.001\right)$, and parallel analysis indicated that three factors should be retained. All items loaded highly on their respective factors, and the three-factor solution explained $70 \%$ of the variance. However, because of the high correlations between the factors after Promax rotation (ranging from 0.65 to 0.74 ) we tested two alternative models in the CFA: one with three orthogonal factors and one with the second order factor measured by the three first order factors. The model with the second-order factor showed significantly better fit $\left(\Delta \chi^{2}(3)=1479.6, p<0.001\right)$, compared to a model with orthogonal factors. Moreover, the model with second order factors had acceptable fit indices $\left(\chi^{2}(24)=142.68\right.$, $p<0.001$, RMSEA $=0.072,95 \%$ CI $[0.061,0.084$, SRMR $=0.028, \mathrm{CFI}=0.979, \mathrm{TLI}=0.968)$, whereas an orthogonal solution provided poor model fit $\left(\chi^{2}(27)=\right.$ $1622.28, p<0.001$, RMSEA $=0.251,95 \%$ CI $[0.240$, $0.261], \mathrm{SRMR}=0.411, \mathrm{CFI}=0.712, \mathrm{TLI}=0.616$ ). Following these results, we computed a mean score from all nine items, which showed high reliability $(\alpha=0.92)$.

\section{Pseudoscientific Information Beliefs}

Both KMO (0.85) and Bartlett's sphericity test $\left(\chi^{2}(10)=\right.$ $5845.99, p<0.001)$ indicated that five items are suitable for factor analysis. However, after the first iteration of the analysis, one item ("alcohol") was excluded from the analysis due to low factor loading. We suspect that this item caused confusion among participants, as alcohol is actually an effective disinfectant and should not be included in the list of pseudoscientific remedies for infection. Next, parallel analysis suggested that one factor should be retained. All items loaded high on one extracted factor, that accounted for $62 \%$ of the 
Table 1 Descriptive statistics and correlations between all variables included in the study

\begin{tabular}{lllllllll}
\hline & $M$ & $S D$ & SE & SI & KI & \multicolumn{2}{l}{$r$} \\
\hline & & & & & & 2 & 3 & 4 \\
(1) COVID-19 conspiracy beliefs & 2.06 & 0.969 & 0.02 & 0.96 & -0.01 & .56 & -.44 & -.46 \\
(2) Pseudoscientific information beliefs & 2.09 & 1.024 & 0.02 & 0.54 & -0.86 & & -.17 & -.25 \\
(3) Trust in government officials & 3.28 & 0.995 & 0.02 & -0.51 & -0.28 & & .47 \\
$\begin{array}{l}\text { (4) Compliance with official COVID-19 } \\
\quad \text { guidelines }\end{array}$ & 3.32 & 0.679 & 0.02 & -1.21 & 0.84 & & & \\
\hline
\end{tabular}

$M$ - mean, $S D$ - standard deviation; SE- standard error of the mean; SI- skewness index; KI- kurtosis index; $r$ Pearson correlation coefficient. Note: $\mathrm{p}$-values for all correlations are $p<0.001$. variance. The single-factor solution was further shown to be appropriate by CFA $\left(\chi^{2}(2)=28.03, p<0.001, \mathrm{RMSEA}=\right.$ $0.118,95 \%$ CI $[0.081,0.158], \mathrm{SRMR}=0.023, \mathrm{CFI}=0.984$, TLI $=0.953$ ). It should be noted that the RMSEA along with its $95 \%$ CI was high and did not indicate a good fit of a model. However, simulation studies have shown that the RMSEA over-penalizes models with few degrees of freedom (Breivik \& Olsson, 2001) and that it shows lower values for models with a larger number of items. Following this finding and considering acceptable values of other indices used, we argue that the low RMSEA value is due to the small number of items included. Therefore, we find the one-factor solution suitable for the items of pseudoscientific beliefs scale. We formed a mean score of four items, which had high internal reliability $(\alpha=0.86)$.

\section{Trust in Government Officials}

Both KMO (0.88) and Bartlett's sphericity test $\left(\chi^{2}(10)=\right.$ $3642.05, p<0.001)$ indicated that five items are suitable for factor analysis. Parallel analysis indicated that only one factor should be retained. All items loaded on one extracted factor, which accounted for $63 \%$ of the variance. The unidimensional structure was further confirmed by CFA, which indicated good model fit $\left(\chi^{2}(5)=29.744, p<0.001, \mathrm{RMSEA}=0.073\right.$, $95 \%$ CI $[0.049,0.099], \mathrm{SRMR}=0.018, \mathrm{CFI}=0.993, \mathrm{TLI}=$
0.989). The mean of five items was computed and showed high internal consistency $(\alpha=0.90)$.

\section{Compliance with Official COVID-19 Guidelines}

Both KMO (0.92) and Bartlett's sphericity test $\left(\chi^{2}(28)=\right.$ 3952.52, $p<0.001)$ indicated that eight items are suitable for factor analysis. Parallel analysis indicated that only one factor should be retained. The first extracted factor explained $54 \%$ of the variance in compliance with the official COVID-19 guidelines, and all items had appropriate loadings. The unidimensional structure was further confirmed by CFA with a good model fit $\left(\chi^{2}(20)=111.69, p<0.001, \mathrm{RMSEA}=0.070,95 \%\right.$ CI [0.057, 0.083], SRMR $=0.027, \mathrm{CFI}=0.978, \mathrm{TLI}=0.969)$. We proceeded by computing a mean score of eight items that had high internal reliability $(\alpha=0.90)$.

\section{Mediation Analysis}

Descriptive statistics and correlations between scales are presented in Table 1.

The results of the mediation analysis are presented in Fig. 1.

The direct effect of COVID-19 conspiracy beliefs on compliance was significant $(\beta=-0.31, B=-0.21, \mathrm{SE}=0.02$, $p<0.001,95 \%$ CI $[-0.25,-0.17])$. In addition to this direct
Fig. 1 Unstandardized regression coefficients of the multiple mediation analysis

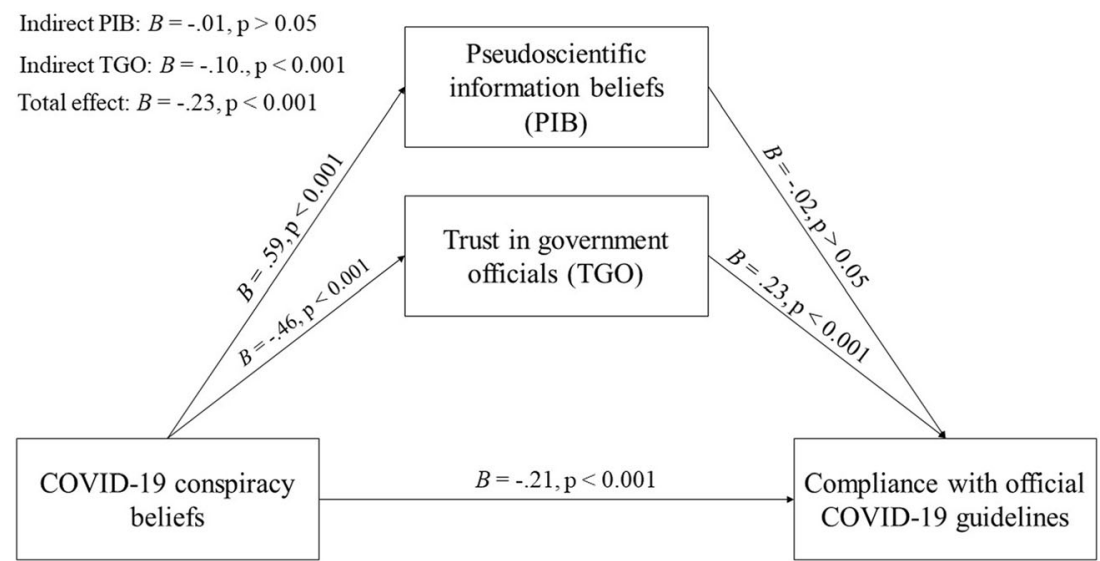


association, results also showed that conspiracy beliefs were indirectly associated with compliance via trust in government officials $(\beta=-0.15, B=-0.10, \mathrm{SE}=0.01, p<0.001,95 \% \mathrm{CI}$ $[-0.12,-0.09])$, with power estimated at $100 \%$. However, an indirect effect via pseudoscientific information beliefs was insignificant $(\beta=-0.02, B=-0.01, \mathrm{SE}=0.01, p>0.05,95 \%$ CI $[-0.03,0.01])$, with an estimated power estimated of $20 \%$. Thus, the association between conspiracy beliefs and compliance with official guidelines is partially mediated by trust in government officials. The direct effect of COVID-19 conspiracy beliefs on the trust in government officials was significant $(\beta=-0.44, B=-0.46, \mathrm{SE}=0.02, p<0.001,95 \%$ CI $[-0.50$, $-0.41]$ ), as was the direct effect of trust in government officials on compliance with official COVID-19 guidelines ( $\beta=$ $0.33, B=0.23, \mathrm{SE}=0.02, p<0.001,95 \%$ CI $[0.19,0.26])$. On the other hand, COVID-19 conspiracy beliefs showed significant direct effect on pseudoscientific information beliefs $(\beta=$ $0.56, B=0.59, \mathrm{SE}=0.02, p<0.001,95 \%$ CI $[0.55,0.63])$, but pseudoscientific information beliefs had no significant direct effect on compliance with official COVID-19 guidelines ( $\beta=$ $-0.02, B=-0.02, \mathrm{SE}=0.02, p>0.05,95 \% \mathrm{CI}[-0.05,0.01])$.

\section{Robustness Analysis}

We further conducted robustness analysis for the relationship between COVID-19 conspiracy beliefs and compliance with official guidelines by including gender, age, and education level as covariates. This analysis was conducted using a smaller sample size $(n=1864)$, because 18 participants did not disclose the information about their gender. Educational level was included in the model as an ordered factor, and we used a diagonally weighted least squares estimator to account for the ordinal nature of this variable. After including the three control variables, the significance of all effects remained the same as in the main analysis. The analysis also revealed that gender had a significant direct effect on adherence to the official COVID-19 guidelines $(\beta=0.26, B=0.38, \mathrm{SE}=0.03$, $p<0.001,95 \%$ CI $[0.32,0.43])$, suggesting that women were more compliant with the guidelines. Similarly, age had a significant direct effect on compliance with guidelines $(\beta=0.07$, $B=0.003, \mathrm{SE}=0.001, p<0.001,95 \%$ CI $[0.0003,0.006]$ ), indicating that older participants report higher levels of compliance. On the other hand, education level had no significant effect on compliance $(\beta=0.01, B=0.008, \mathrm{SE}=0.012$, $p>0.05,95 \%$ CI $[-0.01,0.01])$. In short, after robustness analyses, results remain substantially similar to the patterns displayed in Fig. 1.

\section{Discussion}

In the present study, we sought to replicate and extend previous findings regarding the negative relationship between belief in conspiracy theories about COVID-19 and compliance with official government recommendations and restrictions during the pandemic, and to test whether endorsement of medical misinformation and trust in governmental medical officials would mediate this relationship. The results of the multiple mediation analysis support our hypothesis regarding the direct negative effect of conspiracy belief on adherence to official guidelines. Similarly, the hypothesis regarding the mediating role of trust in government officials is supported. Specifically, COVID-19-related conspiracy beliefs lower levels of compliance with official preventive measures directly and indirectly via lowering trust in government information. The robustness of these findings was further supported in the analysis with several sociodemographic variables as covariates.

The present findings are consistent with previous studies showing that conspiracy beliefs are associated with disregarding public health advice in other health domains (Bogart \& Thorburn, 2005; Grebe \& Nattrass, 2012), and during the current COVID-19 pandemic (e.g., Allington \& Dhavan, 2020; e.g. Biddlestone et al., 2020; Bierwiaczonek et al., 2020; Erceg et al., 2020; Hornik et al., 2021; Kowalski et al., 2020; Latkin et al., 2021; Marinthe et al., 2020; Oleksy et al., 2020). When it comes to the mediating role of trust in government officials in the present study, conspiracy beliefs decreased the level of trust and consequently the level of compliance with governmental protective measures. Marietta and Barker (2018) noted that people who endorse conspiracy theories generally doubt government and scientific information. This makes them prone to disregarding government restrictions and adherence to public safety guidelines. Of course, it could be that people with a generally lower trust in government would actively seek out the contrary and alternative information on how to deal with the crisis, thus showing a greater tendency to believe in conspiracy theories.

This finding highlights the importance of government communication of information about COVID-19 to combat, not only the spread of the coronavirus, but also the spread of misinformation. The current pandemic represents a situation in which it is critical to follow recommendations from trusted scientific sources. At the same time, conspiracy theories are on the rise in social crisis situations like the current pandemic. Not only are they on the rise, but they are also very easy to spread through social media platforms. In fact, studies have shown a positive correlation between COVID-19 conspiracy beliefs and the use of social media as a source of information about the pandemic (Allington et al., 2020). As a result, social networking services such as Facebook, Twitter, and TikTok have come together to help disseminate scientific information by encouraging people to search for trusted sources (Wiederhold, 2020).

Nevertheless, the government has the difficult task of developing strategies to combat the infodemic, which pose a risk 
to public health. Some authors argue that these strategies should include transparency by using e-government tools to share information with the public in real time and more frequently (van Prooijen, 2018). In this context, the Croatian government has been criticized for not providing machinereadable data on coronavirus infections. In response to this criticism, the government published all data, but only two months after the first infected case was detected. Of course, we can only speculate that this and similar examples of governmental decisions led to or reinforced the spread of conspiracy theories, so this is an empirically open question. Another strategy to combat infodemic includes government campaign about fake news related to COVID-19, in which government officials address conspiracy theories as false before they begin to spread too rapidly.

Moreover, consistent with previous studies (e.g., Lamberty \& Imhoff, 2018), we found a positive correlation between beliefs in COVID-19 conspiracy theories and belief in pseudoscientific information about products that can prevent/cure the infection. The importance of including this latter measure in the present study is highlighted in several extreme cases in which people consumed unscientific remedies and suffered serious consequences. Although correlated with both conspiracy beliefs and compliance with the preventive measures, pseudoscientific information beliefs did not significantly predict our main outcome variable in the mediation model. We propose an explanation taking into an account the assumption that individuals would report lower levels of compliance with official protective measures if they used alternative products to prevent infection. However, we did not directly ask participants about their exact actions and use of said products, but rather their belief about product effectiveness. Thus, the participants only expressed their opinions and not their actual behaviour. We propose a possibility that the results would have shown a different pattern if actual behaviour and frequency of use of alternative remedies had been assessed.

Furthermore, mediation analysis showed that women and older participants reported higher levels of compliance with official pandemic guidelines. These results are consistent with previous research showing that women and slightly older participants were more likely to engage in health-protective behaviours (Allington et al., 2020; Franzen \& Wöhner, 2021; Galasso et al., 2020). As for the age effect, one possibility is that older people are more concerned about possible infection and perceive greater risk because symptom severity and mortality rates increase with age. A greater perception of personal risk and serious threat may make older people more likely to follow recommended health guidelines (Marinthe et al., 2020; Plohl \& Musil, 2021). Allington et al. (2020) suggest another plausible explanation noting that older people may be more likely to comply with preventive measures because they use social media less than younger people. Regarding the gender effect, previous studies have also shown that women are more likely to adopt health-protective behaviours, particularly those that can be characterized as non-pharmaceutical, such as handwashing or using face mask (Moran \& Del Valle, 2016). In a similar vein, a recent study found that women reported greater compliance with safety measures such as washing hands and maintaining physical distance in public places (de la Vega et al., 2020). We can speculate on the reasons behind these gender differences. It has been shown that women report greater concern about the pandemic (de la Vega et al., 2020). Moreover, conducting a study on a large sample from eight countries, Galasso et al. (2020) reported large gender differences in the perception of the seriousness of COVID-19, where women were more likely to perceive COVID-19 as a serious health problem. Hence, taking the threat more seriously than men can motivate them to comply with the protective guidelines more. Relatedly, women take fewer risks than men (Harris \& Jenkins, 2006), which might be another reason for greater compliance among women. Altogether, these findings even suggest that lower compliance with health protective measures may play a role in the higher COVID-19 prevalence and mortality rates among men (Sharma et al., 2020).

These findings also suggest some practical implications for policy makers and government communication aimed at reducing the spread of infection. Direct communication with these populations (young people and men) could be beneficial for infection control. Regarding the government communication with the citizens, Zhang et al. (2020) proposed extensive public evidence-based information campaign to increase understanding and knowledge about the COVID-19 disease. In this context, Connolly et al. (2019) emphasized government transparency and use of e-government tools for sharing information with the public as an important aspect of government communication. Furthermore, strategies in fighting the infodemic include providing factual correction for fake news spread via social media, for example in a form of mythbusters officially published by the WHO. Besides providing factual corrective messages to the public, it is also important to focus on encouraging protective behaviour. Therefore, an effective information campaign should also include the benefits of responsible health behaviours.

\section{Limitations and Conclusions}

There are several limitations and implications of this study design that are worth discussing. First, at the time of data collection (second half of May 2020), the spread of coronavirus in Croatia was under control and there were just a few cases indicating local transmission. Future research should investigate the relationship between the variables presented in this study during other phases of the coronavirus pandemic. Second, despite the fairly large sample size in the present study, a representative sample would be more appropriate. 
However, since most conspiracy theories emerge online, we attempted to focus on individuals who are active on social medial. Third, the absence of assessments of other confounding factors, such as personality traits, political ideology, thinking style and cognitive biases, attitudes toward science, and use of social media, can be considered as an additional limitation of the present study. These factors were shown to be related to the conspiracy beliefs (e.g., Alper et al., 2020; Sallam et al., 2021). Therefore, it would be beneficial for future studies to include additional variables (apart from age and gender in the present study) to identify individual characteristics of people who are prone to conspiracy theories. In this way, policy makers can work with more information about target groups when developing specific interventions to combat the pandemic and infodemic.

Despite the limitations described above, this study successfully replicates previous findings and demonstrates the relationship between conspiracy beliefs and adherence to government protective measures. In addition, conspiracy belief was found to lower trust in government information and measures based on scientific epidemiological evidence. This highlights the importance of government's role in developing strategies to combat infodemic.

Overall, the current findings suggest that belief in conspiracy theories can have potentially significant consequences and highlight the need for further research on the role of conspiracy beliefs during the social crisis caused by the COVID-19 pandemic.

Supplementary Information The online version contains supplementary material available at https://oi.org/10.1007/s12144-021-01898-y.

Code Availability Not applicable.

Data Availability The data that support the findings of this study are openly available in Figshare at https://doi.org/10.6084/m9.figshare. 12651371.v1.

\section{Declarations}

Disclosure of Interest there are no relevant financial or non-financial competing interests to report.

\section{References}

Al-Arshani, S. (2020). More than 700 people in Iran have died from trying to use toxic methanol to rid themselves of the novel coronavirus. Business Insider. https://www.businessinsider.com/iraniansdied-from-using-toxic-methanol-cure-coronavirus-2020-4.
Allington, D., \& Dhavan, N. (2020). The relationship between conspiracy beliefs and compliance with public health guidance with regard to COVID-19. Centre for Countering Digital Hate.

Allington, D., Duffy, B., Wessely, S., Dhavan, N., \& Rubin, J. (2020). Health-protective behaviour, social media usage and conspiracy belief during the COVID-19 public health emergency. Psychological Medicine, 1-7. https://doi.org/10.1017/S003329172000224X.

Alper, S., Bayrak, F., \& Yilmaz, O. (2020). Psychological correlates of COVID-19 conspiracy beliefs and preventive measures: Evidence from Turkey. Current Psychology, 1-10. https://doi.org/10.1007/ s12144-020-00903-0.

Atchison, C., Bowman, L. R., Vrinten, C., Redd, R., Pristerà, P., Eaton, J., \& Ward, H. (2021). Early perceptions and behavioural responses during the COVID-19 pandemic: A cross-sectional survey of UK adults. BMJ Open, 11(1), e043577.

Bale, J. M. (2007). Political paranoia v. political realism: On distinguishing between bogus conspiracy theories and genuine conspiratorial politics. Patterns of Prejudice, 41, 45-60. https://doi.org/ 10.1080/00313220601118751.

Belgium Poison Control Center. (2020). The corona crisis is driving the number of calls to the Poison Control Center up sharply. https:// www.antigifcentrum.be/nieuws/coronacrisis-stuwt-aantaloproepen-antigifcentrum-fors-de-hoogte- 0 .

Bentler, P. M. (1990). Comparative fit indexes in structural models. Psychological Bulletin, 107, 238-246. https://doi.org/10.1037/ 0033-2909.107.2.238.

Biddlestone, M., Green, R., \& Douglas, K. (2020). Cultural orientation, powerlessness, belief in conspiracy theories, and intentions to reduce the spread of COVID-19. British Journal of Social Psychology, 59(3), 663-673. https://doi.org/10.1111/bjso.12397.

Bierwiaczonek, K., Kunst, J. R., \& Pich, O. (2020). Belief in COVID-19 conspiracy theories reduces social distancing over time. Applied Psychology: Health and Well-Being, 12(4), 1270-1285. https:// doi.org/10.1111/aphw.12223.

Bogart, L. M., \& Thorburn, S. (2005). Are HIV/AIDS conspiracy beliefs a barrier to HIV prevention among African Americans? JAIDS Journal of Acquired Immune Deficiency Syndromes, 38(2), 213218. https://doi.org/10.1097/00126334-200502010-00014.

Breivik, E., \& Olsson, U. H. (2001). Adding variables to improve fit: The effect of model size on fit assessment in LISREL. In R. Cudeck, S. $\mathrm{Du}$ Toit, \& D. Sörbom (Eds.), Structural equation modeling: Present and future. A Festschrift in honor of Karl Jöreskog (pp. 169-194).

Čavojová, V., Šrol, J., \& Mikušková, E. B. (2020). How scientific reasoning correlates with health-related beliefs and behaviors during the COVID-19 pandemic? Journal of Health Psychology. DOI: https://doi.org/10.1177/1359105320962266.

Connolly, J. M., Uscinski, J. E., Klofstad, C. A., \& West, J. P. (2019). Communicating to the public in the era of conspiracy theory. Public Integrity, 21(5), 469-476. https://doi.org/10.1080/10999922.2019. 1603045.

Croatian Institute of Public Health. (2021). Koronavirus - najnoviji podatci. https://www.hzjz.hr/priopcenja-mediji/koronavirusnajnoviji-podatci/

De La Vega, R., Barquin, R. R., Boros, S., \& Szabo, A. (2020). Could attitudes toward COVID-19 in Spain render men more vulnerable than women? Global Public Health, 15(9), 1278-1291. https://doi. org/10.1080/17441692.2020.1791212.

Dinno, A. (2014). Gently clarifying the application of Horn's parallel analysis to principal component analysis versus factor analysis. Retreived from https://alexisdinno.com/Software/files/PA_for PCA_vs_FA.pdf

Dinno, A. (2018). Paran: Horn's test of principal components/factors. R package version 1.5.2. Retreived from https://CRAN.R-project.org/ package $=$ paran 
Earnshaw, V. A., Eaton, L. A., Kalichman, S. C., Brousseau, N. M., Hill, E. C., \& Fox, A. B. (2020). COVID-19 conspiracy beliefs, health behaviors, and policy support. Translational Behavioral Medicine, 10(4), 850-856. https://doi.org/10.1093/tbm/ibaa09.

Erceg, N., Ružojčić, M., \& Galic, Z. (2020). Misbehaving in the corona crisis: The role of anxiety and unfounded beliefs. Current Psychology. https://doi.org/10.1007/s12144-020-01040-4.

Franzen, A., \& Wöhner, F. (2021). Coronavirus risk perception and compliance with social distancing measures in a sample of young adults: Evidence from Switzerland. PLoS One, 16(2), e0247447. https:// doi.org/10.1371/journal.pone.0247447.

Freeman, D., Waite, F., Rosebrock, L., Petit, A., Causier, C., East, A., Jenner, L., Teale, A. L., Carr, L., Mulhall, S., Bold, E., \& Lambe, S. (2020). Coronavirus conspiracy beliefs, mistrust, and compliance with government guidelines in England. Psychological Medicine, 1-13. https://doi.org/10.1017/S0033291720001890.

Galasso, V., Pons, V., Profeta, P., Becher, M., Brouard, S., \& Foucault, M. (2020). Gender differences in COVID-19 attitudes and behavior: Panel evidence from eight countries. Proceedings of the National Academy of Sciences, 117(44), 27285-27291. https://doi.org/10. 1073/pnas.2012520117.

Gharpure, R., Hunter, C. M., Schnall, A. H., Barrett, C. E., Kirby, A. E., Kunz, J., Berling, K., Mercante, J. W., Murphy, J. L., \& GarciaWilliams, A. G. (2020). Knowledge and practices regarding safe household cleaning and disinfection for COVID-19 prevention United States, may 2020. Morbidity and Mortality Weekly Report, 69, 705-709. https://doi.org/10.15585/mmwr. mm6923e2externalicon.

Grebe, E., \& Nattrass, N. (2012). AIDS conspiracy beliefs and unsafe sex in Cape Town. AIDS and Behavior, 16(3), 761-773. https://doi.org/ 10.1007/s10461-011-9958-2.

Harris, C. R., \& Jenkins, M. (2006). Gender differences in risk assessment: Why do women take fewer risks than men? Judgment and Decision making, 1(1), 48-63. https://doi.org/10.1037/e511092014212.

Horn, J. (1965). A rationale and test for the number of factors in factor analysis. Psychometrika, 30(2), 179-185. https://doi.org/10.1007/ BF02289447.

Hornik, R., Kikut, A., Jesch, E., Woko, C., Siegel, L., \& Kim, K. (2021). Association of COVID-19 misinformation with face mask wearing and social distancing in a nationally representative US sample. Health Communication, 36(1), 6-14. https://doi.org/10.1080/ 10410236.2020 .1847437$.

Imhoff, R., \& Lamberty, P. (2020). A bioweapon or a hoax? The link between distinct conspiracy beliefs about the coronavirus disease (COVID-19) outbreak and pandemic behavior. Social Psychological and Personality Science, 11(8), 1110-1118. https:// doi.org/10.1177/1948550620934692.

Jolley, D., \& Douglas, K. M. (2014a). The effects of anti-vaccine conspiracy theories on vaccination intentions. PLoS One, 9(2), e89177. https://doi.org/10.1371/journal.pone.0089177.

Jolley, D., \& Douglas, K. M. (2014b). The social consequences of conspiracism: Exposure to conspiracy theories decreases intentions to engage in politics and to reduce one's carbon footprint. British Journal of Psychology, 105(1), 35-56. https://doi.org/10.1111/bjop. 12018.

Kowalski, J., Marchlewska, M., Molenda, Z., Górska, P., \& Gawęa, Ł. (2020). Adherence to safety and self-isolation guidelines, conspiracy and paranoia-like beliefs during COVID-19 pandemic in Polandassociations and moderators. Psychiatry Research, 294, 113540. https://doi.org/10.1016/j.psychres.2020.113540.

Lamberty, P., \& Imhoff, R. (2018). Powerful pharma and its marginalized alternatives? Social Psychology, 49(5), 255-270. https://doi.org/10. 1027/1864-9335/a000347.

Latkin, C. A., Dayton, L., Moran, M., Strickland, J. C., \& Collins, K. (2021). Behavioral and psychosocial factors associated with
COVID-19 skepticism in the United States. Current Psychology, 1-9. DOI: https://doi.org/10.1007/s12144-020-01211-3.

Marietta, M., \& Barker, D. C. (2018). Conspiratorial thinking and dueling fact perceptions. In J. E. Uscinski (Ed.), Conspiracy theories and the people who believe them (pp. 214-225). Oxford University Press. https://doi.org/10.1093/oso/9780190844073.003.0014.

Marinthe, G., Brown, G., Delouvée, S., \& Jolley, D. (2020). Looking out for myself: Exploring the relationship between conspiracy mentality, perceived personal risk and COVID-19 prevention measures. British Journal of Health Psychology, 25(4), 957-980. https://doi. org/10.1111/bjhp.12449.

Moran, K. R., \& Del Valle, S. Y. (2016). A meta-analysis of the association between gender and protective behaviors in response to respiratory epidemics and pandemics. PLoS One, 11(10), e0164541. https://doi.org/10.1371/journal.pone.0164541.

Oleksy, T., Wnuk, A., Maison, D., \& Łyś, A. (2020). Content matters. Different predictors and social consequences of general and government-related conspiracy theories on COVID-19. Personality and Individual Differences, 168, 110289. https://doi. org/10.1016/j.paid.2020.110289.

Plohl, N., \& Musil, B. (2021). Modeling compliance with COVID-19 prevention guidelines: The critical role of trust in science. Psychology, Health \& Medicine, 26(1), 1-12. https://doi.org/10. 1080/13548506.2020.1772988.

Pummerer, L., Böhm, R., Lilleholt, L., Winter, K., Zettler, I., \& Sassenberg, K. (2021). Conspiracy theories and their societal effects during the COVID-19 pandemic. Social Psychological and Personality Science, 19485506211000217. https://doi.org/10.1177/ 19485506211000217.

R Core Team. (2020). R: A language and environment for statistical computing. R Foundation for Statistical Computing.

Revelle, W. (2019). psych: Procedures for Personality and Psychological Research, Northwestern University, Evanston, Illinois, USA. Retreived from: https://CRAN.R-project.org/package=psych Version $=1.9 .12$.

Romer, D., \& Jamieson, K. H. (2020). Conspiracy theories as barriers to controlling the spread of COVID-19 in the US. Social Science \& Medicine, 263, 113356. https://doi.org/10.1016/j.socscimed.2020. 113356.

Rosseel, Y. (2012). Lavaan: An R package for structural equation modeling. Journal of Statistical Software, 48(2), 1-36. https://doi.org/10. 18637/jss.v048.i02.

Sallam, M., Dababseh, D., Eid, H., Al-Mahzoum, K., Al-Haidar, A., Taim, D., Yaseen, A., Ababneh, N. A., Bakri, F. G., \& Mahafzah, A. (2021). High rates of COVID-19 vaccine hesitancy and its association with conspiracy beliefs: A study in Jordan and Kuwait among other Arab countries. Vaccines, 9(1), 42. https://doi.org/10. 3390/vaccines 9010042 .

Schoemann, A. M., Boulton, A. J., \& Short, S. D. (2017). Determining power and sample size for simple and complex mediation models. Social Psychological and Personality Science, 8(4), 379-386. https://doi.org/10.1177/1948550617715068.

Sharma, G., Volgman, A. S., \& Michos, E. D. (2020). Sex differences in mortality from COVID-19 pandemic: Are men vulnerable and women protected? JACC: Case Reports, 2(9), 1407-1410. https:// doi.org/10.1016/j.jaccas.2020.04.027.

SimilarWeb. (2020, June, 1). Top Websites Ranking. Top sites ranking for News And Media in Croatia. https://www.similarweb.com/topwebsites/croatia/category/news-and-media/

Steiger, J. H. (1990). Structural model evaluation and modification: An interval estimation approach. Multivariate Behavioral Research, 25, 173-180. https://doi.org/10.1207/s15327906mbr2502_4.

Tabachnick, B. G., \& Fidell, L. S. (2012). Using multivariate statistics. Pearson.

Teovanović, P., Lukić, P., Zupan, Z., Lazić, A., Ninković, M., \& Žeželj, I. (2020). Irrational beliefs differentially predict adherence to 
guidelines and pseudoscientific practices during the COVID-19 pandemic. Applied Cognitive Psychology, 1-11. DOI: https://doi.org/ 10.1002/acp.3770.

Tucker, L. R., \& Lewis, C. (1973). A reliability coefficient for maximum likelihood factor analysis. Psychometrika, 38, 1-10. https://doi.org/ 10.1007/BF02291170.

van Prooijen, J. W. (2018). Empowerment as a tool to reduce belief in conspiracy theories. In J. E. Uscinski (Ed.), Conspiracy theories and the people who believe them (pp. 432-442). Oxford University Press. https://doi.org/10.1093/oso/9780190844073.003.0030.

van Prooijen, J. W., \& Douglas, K. M. (2017). Conspiracy theories as part of history: The role of societal crisis situations. Memory Studies, 10(3), 323-333. https://doi.org/10.1177/1750698017701615.

Wiederhold, B. K. (2020). Social media use during social distancing. Cyberpsychology, Behavior and Social Networking, 23(5), 275276. https://doi.org/10.1089/cyber.2020.29181.bkw.

Wood, M. J. (2018). Propagating and debunking conspiracy theories on twitter during the 2015-2016 Zika virus outbreak. Cyberpsychology, Behavior and Social Networking, 21(8), 485490. https://doi.org/10.1089/cyber.2017.0669.
World Health Organization. (2021a). Coronavirus disease (COVID-19): Weekly Epidemiological Update. https://www.who.int/ publications $/ \mathrm{m} /$ item/weekly-epidemiological-update-on-covid-19\% 2D\%2D-20-april-2021

World Health Organization. (2021b). Coronavirus disease (COVID-19) advice for the public: Mythbusters. https://www.who.int/ emergencies/diseases/novel-coronavirus-2019/advice-for-public/ myth-busters

Yan, A. (2020, February 15). Coronavirus: Desperate times drive some Chinese people to take desperate measures. South China Morning Post. https://www.scmp.com/news/china/society/article/3050673/ coronavirus-desperate-times-drive-some-chinese-people-take

Zhang, X., Wang, F., Zhu, C., \& Wang, Z. (2020). Willingness to selfisolate when facing a pandemic risk: Model, empirical test, and policy recommendations. International Journal of Environmental Research and Public Health, 17(1), 197. https://doi.org/10.3390/ ijerph17010197.

Publisher's Note Springer Nature remains neutral with regard to jurisdictional claims in published maps and institutional affiliations. 